\title{
Introduction to 2019 ACM-BCB Highlights Session
}

\author{
Jianlin Cheng \\ Department of Electrical Engineering and Computer Science \\ University of Missouri \\ Columbia, MO 65211, USA \\ chengji@missouri.edu
}

\begin{abstract}
2019 ACM-BCB highlights session is comprised of five significant advances in protein structure prediction, protein function analysis, and genomics data analysis that were recently published in several reputable journals in the field including Proteins, PLoS Computational Biology, Genome Research, and Journal of Mathematical Biology. In protein structure prediction, "Protein tertiary structure modeling driven by deep learning and contact distance prediction in CASP13" [1] reports the latest advance of applying deep learning and contact distance prediction to significantly improve both template-based and template-free protein structure prediction; and "How effective is contact-assisted protein threading?" [2] describes a new approach of using contact prediction to improve the templatebased protein structure prediction (i.e. protein threading). In protein function analysis, "CoPhosK: a method for comprehensive kinase substrate annotation using cophosphorylation analysis" [3] discusses a new method called CoPhoSK that uses co-phosphorylation analysis on mass spectrometry data to predict substrates of protein kinases. In genomics data analysis, "Beta-binomial modeling of CRISPR pooled screen data identifies target genes with greater sensitivity and fewer false negatives" [4] presents a new method called CRISPRBetaBinomial that applies Beta Binomial model to recognize target genes from CRISPR pooled screen data; and "Low-dimensional representation of genomic sequences" [5] describes a multilateration-based embedding method for representing genomic sequences (e.g. DNA sequences) for classification and clustering analysis. These selected works have both significant technological innovations and valuable applications and therefore are appealing to the broad community of bioinformatics and computational biology.
\end{abstract}

\section{CCS CONCEPTS}

- deep learning $\bullet$ bioinformatics $\bullet$ machine learning

\section{KEYWORDS}

deep learning, protein structure prediction, genomics, CRISPR

\section{ACM Reference format:}

Jianlin Cheng. 2019. Introduction to 2019 ACM-BCB Highlights Session. In Proceedings of the $10^{\text {th }}$ ACM Conference on Bioinformatics, Computational Biology, and Health Informatics (ACM BCB'19), Spet. 7-10, Niagara Falls, NY, USA. ACM, New York, New York, 1 page. https://doi.org/10.1145/3307339.3342619

\section{ACKNOWLEDGMENTS}

The work is supported by the National Natural Science Foundation (NSF) under two grants (Grant No.: DBI1759934 and IIS1763246) and an NIH grant (Grant No. R01GM093123).

\section{REFERENCES}

[1] Jie Hou, Tianqi Wu, Renzhi Cao, Jianlin Cheng. 2019. Protein tertiary structure modeling driven by deep learning and contact distance prediction in CASP13. Protein. In press. https://doi.org/10.1002/prot.25697

[2] Sutanu Bhattacharya, Debswapna Bhattacharya. Does inclusion of residue residue contact information boost protein threading? 2019. Proteins. In press.

[3] Marzieh Ayati, Danica Wiredja, Daniela Schlatzer, Sean Maxwell, Ming Li, Mehmet Koyutürk, Mark R. Chance. 2019. CoPhosK: A method for comprehensive kinase substrate annotation using co-phosphorylation analysis. 2019. PLoS Computational Biology. In press.

[4] Hyun-Hwan Jeong, Seon Young Kim, Maxime W.C. Rousseaux, Huda Y. Zoghbi and Zhandong Liu. 2019. Beta-binomial modeling of CRISPR pooled screen data identifies target genes with greater sensitivity and fewer false negatives. In press.

[5] Richard C. Tillquist, Manuel E. Lladser. 2019. Low-dimensional representation of genomic sequences. In press.
Permission to make digital or hard copies of part or all of this work for personal or classroom use is granted without fee provided that copies are not made or distributed for profit or commercial advantage and that copies bear this notice and the full citation on the first page. Copyrights for third-party components of this work must be honored. For all other uses, contact the Owner/Author.

ACM-BCB '19, September 7-10, 2019, Niagara Falls, NY, USA.

(c) 2019 Copyright is held by the owner/author(s)

ACM ISBN 978-1-4503-6666-3/19/09.

DOI: https://doi.org/10.1145/3307339.3342619 